\title{
Un nuevo horizonte para una medicina legal más social. El médico forense como garante de los derechos fundamentales de las personas ( parte I I)
}

\author{
A new horizon for a more social Legal Medicine. The forensic physician \\ as guarantor of the fundamental rights of the people (Part II)
}

\begin{abstract}
Resumen
Se reflexiona sobre el papel actual del médico, y en particular del médico legista, como garante de los derechos fundamentales de la persona en la sociedad actual. En la segunda mitad del siglo XX cambia el paradigma que habia regulado la relación medico/paciente, pasando de estar regida por el principio de beneficencia al de autonomia. Ello genera situaciones especialmente complejas para el acto médico, sobretodo en el final de la vida. Se analiza la encrucijada en la que se ve el médico funcionario al defender los intereses de sus pacientes en detrimento de los de la Administración que le paga, especialmente en tiempos de recesión económica como los presentes. Se exponen numerosos casos en los que el médico forense se puede ver coaccionado para no realizar una autopsia u otra diligencia judicial, o bien se ve involucrado en decisiones de otros, generalmente de los jueces, achacadas al médico forense. Igualmente se consideran casos en los que los derechos fundamentales de los ciudadanos están siendo vulnerados por los Estados.

Se plantea la necesidad de establecer la Medicina Legal en el ámbito hospitalario, como una especialidad médica en pie de igualdad con las demás especialidades, en relación con la gran trascendencia jurídica, penal, civil, administrativa y económica del parte de lesiones y otras actuaciones de la asistencia hospitalaria. Se cuestiona si siempre la formación de los médicos forenses es la adecuada para alcanzar unos niveles de garantía de calidad en su trabajo, especialmente en la práctica de la autopsia, una operación de cuyo resultado dependen tantos derechos y responsabilidades y que no puede ser rehecha. También se tratan los nuevos desafíos que afronta el médico legista como la Bioética y los problemas medicolegales relacionados con el aborto, el libre comercio en el trasplante de órganos o la eutanasia voluntaria. Todo ello desde la perspectiva de la defensa de los derechos de los ciudadanos.
\end{abstract}

Palabras clave: Medicina Legal. Médico forense. Relación médico/paciente. Autonomía del paciente. Garante de los derechos fundamentales de las personas.

\section{Abstract}

The current role of the physician and in particular the forensic physician as guarantor of the fundamental rights of person in today's society is reviewed in this article. The special importance of the change in the second half of the twentieth century in the doctor / patient relationship by which the principle of beneficence to autonomy gave way, creating complex situations especially when persons are at the end of life. The crossroads where the medical officer is to defend the interests of their patients at the expense of the Administration that pays him, especially in times of economic recession as present, are analyzed. There are numerous cases in which the forensic pathologist may be forced not to perform an autopsy or other judicial proceedings, or is involved in decisions taken by others, judges generally, and then blamed to de forensic physician. We also consider cases in which the fundamental rights of citizens may be violated by the State.

The need for Legal Medicine in hospitals, as a medical specialty equal to other specialties is growing, related to the increasing great legal importance of criminal, civil, administrative and economical of injuries, as well as other actions that arise during hospital care. It is also questioned whether training of forensic physicians is adequate to achieve levels of quality assurance in their work, especially in the practice of autopsy, an operation on wich results depend many rights and obligations and that can not be redone. The are also new challenges facing as forensic physician: the Bioethics and medicolegal problems regarding abortion, free trade in organ transplantation or voluntary euthanasia, issues that are also discussed. These topics are reviewed from the perspective of protecting the rights of the citizens.

Key words: Legal Medicine. Forensic physician. Doctor/patient relationship. Patient autonomy. Guarantor of the fundamental rights of persons.

\section{E. Villanueva Cañadas ${ }^{1}$ \\ V. Ramos Medina ${ }^{2}$ \\ H. Villanueva de la Torre $^{3}$}

${ }^{1}$ Catedrático Emérito de Medicina Legal y Forense. Universidad de Granada.

${ }^{2}$ Médico Forense.

Jefe de Sección

de Histopatología

Forense. Instituto de

Medicina Legal

de Málaga.

${ }^{3}$ Médico Forense. Instituto de Medicina Legal de Málaga.

Correspondencia:

Enrique Villanueva Cañadas Facultad de Medicina. Avda. de Madrid, 11. 18071 Granada.

E-mail:

guadalfe040@telefonica.net

Fecha de recepción:

10. OCT. 2013

Fecha de aceptación:

17. OCT. 2013 
En la primera parte de este artículo hemos querido plantear una serie de cuestiones que nos llevan a un territorio muy fértil para nuestra especialidad. Ello supone aflorar una serie de cuestiones que demuestran palmariamente que la Medicina Legal no se agota en la Patología Forense; existe una Medicina Legal más allá de los juzgados y con una gran proyección social. Pero para alcanzar estos objetivos hemos de situar a los forenses, especialistas en Medicina Legal y Forense, en los lugares en que se producen los acontecimientos: los hospitales. Esto no es una ocurrencia de fin de semana; hay países, más modernos que el nuestro, y más avanzados, al menos en lo que a la Medicina Forense se refiere, como Australia, en donde ya se produce $\mathrm{e}^{10,11}$

Desde la presidencia de la Academia Internacional de Medicina Legal, uno de nosotros (E. Villanueva) viene reclamando desde 1992 el reconocimiento mundial de la especialidad de Medicina Legal como una especialidad médica en pie de igualdad con las otras especialidades hermanas, y una de las bases para esta reclamación es justamente la defensa de los ciudadanos en el disfrute pleno de sus derechos, desde una perspectiva más amplia que la contenida en la carta de los derechos del paciente, que va desde la justa evaluación de unas lesiones de tortura, la correcta identificación de personas, la realización de una exploración completa y experta de una mujer violada o maltratada, hasta el derecho que asiste a cualquier acusado de contar con una pericia independiente, la correcta evaluación del daño corporal por las lesiones sufridas en cualquier tipo de accidentes, o el reconocimiento de una enfermedad profesional, sin que ello dependa de su nivel económico. Al mismo tiempo, el derecho que asiste a todos los ciudadanos de ser resarcidos por los daños producidos por el funcionamiento normal o anormal de las administraciones públicas, contando para ello con un sistema médico legal que permita emprender este tipo de acciones con garantías.

La casuística es muy amplia y abarca muchos y delicados temas, como la mala praxis (en la cual los pacientes se ven en serias dificultades para encontrar un perito cualificado que vaya contra otro médico), los casos de consentimiento por representación, las causas de incapacitación civil, en definitiva, situaciones en las que están en juego derechos e intereses. Se nos dirá que eso ya se hace, pero lo que aquí se pretende es dar un paso más, tanto cualitativo como cuantitativo.

La Medicina Legal nace con el parte de lesiones en la Constitutio Criminales Carolina (1532) ${ }^{12}$, modesto documento por el cual todo médico que asista a un lesionado o intervenga en cualquier otro proceso médico que pudiera ser constitutivo de delito tiene el deber legal de comunicarlo al juez. Esta obligación cobra cada vez mayor relevancia, no sólo penal, sino social y económica. El modesto documento puede ser el único que sustente una reclamación millonaria. Cualquier violencia contra las personas siempre pasa por las manos de un médico, de urgencias primero y legista después. A veces es el único documento en el que se basará una sentencia en la que pueden estar en juego muchos intereses, tanto penales como civiles. ¿Son realmente conscientes los médicos que actúan en una urgencia de la trascendencia que tiene, en tanto defensa de los derechos fundamentales, la realización de un parte de lesiones? ¿Y el médico forense que realiza un parte de sanidad, cuyo contenido será el que el juez asuma en un 95\% de los casos? ¿Nos lo estamos planteando en términos de garantes de los derechos de los ciudadanos y como pruebas preconstituidas que no se podrán reproducir? (La prueba preconstituida es aquella prueba que existe antes de la apertura del proceso judicial, y que está a disposición del juez en cualquier momento; es sobre todo una prueba documental. No confundir con la prueba anticipada, que ésta sí se realiza una vez incoado un procedimiento.) A diario nos lamentamos de las imprecisiones semánticas o conceptuales vertidas en un parte de lesiones. Una palabra, por ejemplo edema traumático de retina, deslizada en un parte de lesiones por un médico de urgencias o por un clínico, que en ese momento no atisba la trascendencia del término usado, puede a la postre acarrear la tipificación de un delito de lesiones grave.

Las autopsias, tanto las clínicas como la judiciales, son el otro gran dique de contención de muchas injusticias y atropellos por parte del Estado y la Administración. Una autopsia mal hecha no puede rehacerse, y con ella se escaparán la verdad y la posibilidad de cualquier justicia. Cuando una sentencia emitida por un Juez de Instrucción en un caso de valoración de daño corporal dice: jurisprudencialmente en relación con los informes médicos oficiales, se considera que los Médicos Forenses llevan a cabo sus informes de forma imparcial y profesional, de manera que no existe razón alguna para dudar de su veracidad, cuando realizan sus cometidos profesionales, teniendo los Informes que prestan un alto poder convictivo, en cuanto que no existe elemento subjetivo alguno para dudar de tal veracidad, precisamente en función a la imparcialidad y profesionalidad que caracteriza su cometido en los entes públicos. Esta opinión, que como dice la sentencia nace de la jurisprudencia, debería hacernos reflexionar en dos direcciones. Yo no creo que los forenses, aunque resulte tan halagador el comentario de esta sentencia, confundan imparcialidad con 
infalibilidad. A veces, incluso, hay sobradas razones para dudar de su profesionalidad y de la veracidad de sus actuaciones, entendidas ambas no en tanto a que lo que dicen se ajusta al criterio que el forense tiene de ese caso, sin ser mediatizado por nadie (imparcialidad), sino a que el contenido de su pericia se ajuste a la verdad científica y no pueda ser falseada, en el sentido popperiano, por otro. (El falsacionismo o principio de falsabilidad es una corriente epistemológica propuesta por el filósofo austríaco Karl Popper, para quien la experiencia no es capaz de probar que una teoría es verdadera; lo único que la experiencia puede probar es que una hipótesis científica es falsa).

¿Responden los forenses titulares y titulados especializados de la profesionalidad de los interinos, cuya cualificación profesional nadie ha contrastado? ¿Puede predicarse de todos ellos que responden a este criterio universal de competencia profesional? ¿Es una autopsia un acto tan sencillo e inocuo para confiarla a cualquiera? Causa cuanto menos preocupación cómo se confía la realización de esta diligencia a personas que no consta que tengan la cualificación necesaria. La realidad es que el patólogo, en este caso de la autopsia judicial, está revestido de una autoridad científica que nace de su posición en el proceso, como perito oficial de quien no se duda; sus decisiones no serán cuestionadas y, si lo fueran, gozarán del mismo carisma que la verdad notarial, y nadie las va a destruir. A veces creo que nos hemos quedado sólo con la complacencia de que nuestra pericia es indestructible, sin pensar en la enorme responsabilidad que asumimos precisamente por ello. Somos cómplices, al no revelar que una práctica, que no puede rehacerse, de la que dependen tantos derechos y tantas obligaciones, no se puede confiar a cualquiera, como desgraciadamente ocurre con más frecuencia de la deseada. Creo que éste sería el mejor paradigma de cómo el forense puede defender los derechos fundamentales de los ciudadanos. Parafraseando las palabras que figuran en el frontispicio del Teatro Anatómico de Padua, Hic est locus ubi mors gaudet succurrere vitae (Éste es el lugar donde la muerte se complace de ayudar a la vida), en el frontispicio de las salas de autopsias de la IMLs deberíamos escribir Éste es el lugar sagrado donde los médicos forenses trabajan para velar por tus derechos. 0 tener presente lo que se lee en el Ontario Forensic Pathology Service de Canadá: The dead have his rights as the living have; injustice to them is one of the worst forms of all injustice (Thomas D'Arcy McGee). (Los muertos, como los vivos, tienen sus derechos, la injusticia con ellos es una de las peores formas de injusticia).

De otra parte, el progreso técnico ha puesto en manos de los médicos un enorme poder, que llega a esta- blecer quién vive y quién no, quién nace, y cómo y cuándo se muere. Aquí están representados los máximos derechos de los ciudadanos. La protección de la vida y ser el albacea leal que administra el principio de beneficencia con rigor y siempre en beneficio del paciente, en los momentos más trascendentes del ser humano, como es el de vivir su muerte.

El consentimiento por representación, generalmente bien enunciado, está mal regulado, por su inconcreción, en la Ley 41/2002 ${ }^{13}$; llegado el momento de los estados límites, se presenta como un dilema para el médico, desde su posición de garante ${ }^{14}$.

Sería conveniente reflexionar sobre estas cuestiones y que los médicos tomasen postura sobre ellas, ya que cada vez caminan más hacia una deriva legal: el juez decide, en detrimento de una postura clínica y ética, más en consonancia con el respeto a la voluntad del paciente.

Pero la cuestión capital está en la necesidad de una reforma profunda de los sistemas de organización forense, no sólo en España sino en el mundo, que junto a una Medicina Forense de calidad contrastada permita una independencia de criterios y de actuación. Hablar de defensa de derechos humanos con una medicina legal oficial intervenida es un sarcasmo.

Algunos países han resuelto esta cuestión desde hace muchos años de modo satisfactorio; otros creen que la tienen resuelta, entre otras cosas porque no hay sistemas de controles externos; otros, como nosotros o incluso los poderosos y avanzados Estados Unidos de América, no lo han hecho. La Prof. Castellano ${ }^{15}$, en su discurso de ingreso en la Real Academia Nacional de Medicina (20 de mayo de 2012), hacía alusión a esta cuestión, y ya el título del discurso es suficientemente clarificador: La construcción de la Medicina Legal y Forense en España: después de ciento setenta años, una tarea inacabada. Diversas instituciones académicas norteamericanas han publicado un informe, Strengthening forensic science in the United States: A path forward ${ }^{16}$, en el cual se denuncia la situación, en muchos casos caótica, de la Medicina Legal americana, en la que conviven sistemas casi medievales (coroner) con los institutos forenses más modernos del mundo ${ }^{17-19}$.

"Los sistemas del Medical Examiner/Coroner (ME/C) funcionan con variables niveles de experiencia, a menudo con deficiencias en instalaciones, equipos, personal, educación y formación. Por desgracia, la mayoría de los sistemas tienen bajo presupuesto y están escasos de personal. Al igual que con otros campos de las ciencias forenses, no hay títulos o certificaciones nacionales obligatorias necesarios 
para los investigadores de la muerte. Tampoco la experiencia médica es siempre necesaria (El Coroner puede no ser ni médico). Además, no hay un reconocimiento conjunto de estándares de desempeño o mejores prácticas para los sistemas $M E / C$, ni existen incentivos para poner en práctica un método conjunto. También hacen falta métodos de control de calidad o garantía de calidad. Está claro que la conversión de los sistemas de Coroner al de Medical Examiner, según lo recomendado por muchos estudios, se ha detenido en esencia y requiere incentivos federales para seguir adelante. La Ley de 1954, modelo de examen post mórtem, debe ser revisada y actualizada para incluir los elementos de una ley progresista y sensible en la investigación de la muerte".

Finalmente hemos de señalar una nueva amenaza: la Bioética. Esta nueva disciplina, que no ciencia, está de moda en el mundo, y algunos nos hemos refugiado en ella para decir cosas que desde la Medicina Legal no tienen eco, pero sí hemos de ser conscientes de que muchas cuestiones que hoy se plantean como de Ética o Bioética caen de lleno en el campo de la Medicina Legal. La experiencia nos dice que reclamar las cosas como si se tratara de un derecho patrimonial sobre ellas carece de eficacia. Hoy los derechos históricos sobre tal o cual disciplina sólo pueden reclamarse desde la competencia profesional de aquellos que la practican. Creemos que el Médico Forense, por su posición legal y por su formación profesional, sería la persona más idónea para garantizar los derechos fundamentales conculcados o la seguridad de los ciudadanos. Como ejemplo valgan los tres siguientes.

Asistimos con enorme preocupación a la aceptación social de ciertas posturas liberales extremas que abogan por admitir un libre comercio de órganos, la eutanasia voluntaria o el libre derecho a abortar, concebidos todos ellos como derechos colectivos. Son asuntos con un gran trasfondo ético, pero sobre todo y ante todo son medicolegales. Al fin son delitos (amputación de miembro principal, homicidio, aborto) y por tanto materia de nuestra competencia.

En nuestras manos está la solución a muchas cosas que hoy nos pueden parecer utópicas, pero nadie sabe de violencia más que los médicos legistas y desde nuestra atalaya privilegiada hemos de iluminar al mundo, pero sobre todo a nuestros compañeros médicos, que se baten en las trincheras de la medicina del día a día.

Un día no muy lejano se aceptó sin rechistar, sin someter siquiera a la discusión científica, que la muerte cerebral equivalía a la muerte legal. Entonces las razones esgrimidas eran de simple utilitarismo: había que salvar los trasplantes de órganos vitales, había que salvar al Dr. Barnard y al Dr. Martínez Bordiu. No nos quejamos entonces y no nos quejamos ahora, pero hemos de admitir que, con la excepción de Hans Jonas ${ }^{20}$, muy pocos intelectuales desearon abrir un debate impopular. Hoy hemos ampliado el horizonte de la clásica muerte legal a una muerte por la confirmación del cese irreversible de las funciones circulatorias y respiratorias, o del cese irreversible de las funciones encefálicas.

No es este el momento ni el lugar para entrar a discutir un decreto, el RD 2070/99, que estuvo en vigor hasta el 18 de enero de 2013 (derogado por el RD 1723/2012 de 28 de diciembre). Para los casos de muertes violentas se adoptan algunas cautelas, y se hace referencia directa a la autorización judicial. Así, en el artículo 9, apartado 5, se recogen las condiciones y requisitos para la extracción de órganos de fallecidos:

“... en los casos de muerte accidental, así como cuando medie una investigación judicial, antes de efectuarse la extracción de órganos deberá recabarse la autorización del juez que corresponda, el cual, previo informe del médico forense, deberá concederla siempre que no se obstaculice el resultado de la instrucción de las diligencias penales.

"En los casos de muerte por parada cardiorrespiratoria que requieran autorización judicial para proceder con las maniobras de mantenimiento de viabilidad de los órganos y con las maniobras de preservación se actuará de conformidad con lo establecido en el anexo l."

Tras 15 minutos sin respuesta del juzgado prosiguen con la conservación para la extracción.

Evidentemente prima el interés del trasplante sobre el derecho de los ciudadanos a expresar su voluntad. ¿Dónde estaba escrita la necesidad de consultar las voluntades anticipadas de esta persona, sobre si querían ser o no donantes, antes de la reforma de la ley? Sería aceptable una expropiación del cadáver a los familiares, pero no lo es al individuo, al titular del derecho, al menos mientras no esté legalmente muerto. Otra consecuencia del progreso técnico ha sido el cuándo y el cómo se muere, que también pueden ser modificados a voluntad del médico. Con ello se plantea por primera vez algo insólito: iEl derecho a morir! ${ }^{21}$. Entre los derechos de la personalidad no figura éste, sólo su opuesto: el derecho a la vida. Aunque la vida se nos concede al margen de la sociedad, por un hecho natural, el ser humano demanda de inmediato a su entorno y a sus semejantes, más que ningún otro ser vivo, una serie de exigencias sin las cuales sucumbiría, demandas que 
podrían ser o no otorgadas. La sociedad se echa sobre sus espaldas una obligación: la protección de la vida del otro. Ciertamente que el derecho natural es a la vida, pero la protección de ésta, como correlato, ya es de contrato social. El derecho a la protección es un derecho legal y, por tanto, regulable. El derecho a la vida es el primer y más relevante derecho de la personalidad, el que da soporte a los demás. ¿Qué da el individuo a cambio de esta protección? Entendemos que los individuos contraen con la sociedad una obligación, que consistiría en esforzarse en preservarla al menos en la misma medida en que se lo exigimos a los demás, el correlativo al derecho a la vida sería la obligación de vivirla. No sería coherente que yo exija a la sociedad que vele por mi vida, e incluso que se la castigue si no lo hace (Art 195 CP: omisión del deber de socorro), mientras yo no lo hago. La muerte, sin embargo, es algo negativo; la persona no obtiene ningún bien de ella, vendrá cuando el destino lo establezca, de ahí que no sea necesaria la protección para morir cuando se dan las circunstancias para ello, ni que sea necesario demandar un derecho a morir. Pero cuando el curso natural de la vida se altera y este devenir se interrumpe de modo artificial, alterando lo que se denomina ortotanasia, para caer en la distanasia, entonces sí que el ser humano tiene derecho a exigir un derecho a morir. Evidentemente existe un derecho a morir, un derecho que no debe ser violentado ni expropiado, y que como todo derecho de la personalidad es personalísimo. Este derecho a morir tiene una peculiaridad que lo diferenciaría de los otros, y es que habría que enunciarlo en términos negativos. La muerte no me pertenece, lo que realmente me pertenece es que no se altere el curso natural de ella sin mi consentimiento. Es el acto de morir el que realmente me pertenece y es el que tiene que estar protegido de injerencias externas.

Fijar el momento de la muerte, la data de la muerte, desde la perspectiva medicolegal no puede dejarse a la indefinición o a criterios más o menos laxos. Anudada a ella hay muchos derechos de la personalidad en disputa. La muerte pone fin a la personalidad jurídica; el momento de la muerte queda fijado por el certificado de defunción. La data de la muerte es relevante para muchos negocios jurídicos, algunos relevantes, como es la declaración de herederos en aquellos casos de dos personas llamadas a sucederse, y el vivo hereda al muerto. Salvo lo dispuesto para la conmoriencia (Art 33 del CC: "Si se duda, entre dos o más personas llamadas a sucederse, quién de ellas haya muerto primero, el que sostenga la muerte anterior de una o de otra debe probarla; a falta de prueba, se presumen muertas al mismo tiempo y no tiene lugar la transmisión de derechos de uno a otro"), pueden plantearse otros casos en los que se prolonga artificialmente la vida de una persona de modo interesado, no para el muriente sino para los intereses de otros. Desde el punto de vista legal, las cuestiones más graves se plantean en la esfera penal, cuando hay que decidir si se interrumpen las medidas extraordinarias de soporte o cuando se plantea lo contrario, si es necesario establecer unas medidas extraordinarias o no, y si el paciente tiene derecho a rechazar determinados tratamientos.

Cuando todas estas cosas suceden, ¿dónde estamos nosotros los forenses? ¿Merece la pena realmente incrementar nuestra ya difícil tarea con más problemas? La respuesta debe producirse y pronto, si no queremos que otros, con menos conocimientos y carácter moral, la usurpen y ocupen nuestro lugar. Llevamos luchando muchos años por conseguir que la Medicina Legal sea una especialidad médica como cualquier otra. Deseamos una Medicina Legal con horizontes más amplios y con mayores compromisos sociales. $A$ veces, entre nosotros, nos preguntamos " $i$ Es que nos explicamos tan mal que nadie nos entiende?". Creemos que más bien sucede lo que decía Ludwig Wittgenstein al encontrar en los filósofos vieneses, y después en Inglaterra, una gran incomprensión de sus teorías: "¿Pero acaso no sentimos que quien no ve allí un problema está ciego ante algo importante...? ¿No me gustaría acaso decir que ese tal vive precisamente ciego, como un topo, y que si pudiera ver, vería el problema?"22. Rectificamos al sabio y rebelde maestro, no si pudiera ver, sino si quisiera ver. Y el problema no es, desgraciadamente, sólo de los españoles, sino mucho más generalizado, como se deja traslucir por el Documento de la Academia Americana.

Hoy expresamos aquí el amor por esta disciplina, a la que hemos dedicado nuestra vida, y estamos tan convencidos de ella que a veces podría ser una idea sobrevalorada, iy por tanto patológica!

Los autores declaran no tener conflicto de intereses. 


\section{Bibliografía}

10. Curran J, McD Taylor D. National Coroners Information System: a valuable source of lessons for emergency medicine. Emerg Med Australas. 2012;24(4):442-50.

11. Bedford PJ. Routine CT scan combined with preliminary examination as a new method in determining the need for autopsy. Forensic Sci Med Pathol. 2012;8(4):390-4.

12. Corbella J. En: Gisbert Calabuig JA, Villanueva Cañadas E, editores. Gisbert Calabuig. Medicina legal y toxicología. $6^{a}$ ed. Barcelona: Masson; 2004.

13. Ley $41 / 2002$, de 14 de noviembre, básica reguladora de la autonomía del paciente y de derechos y obligaciones en materia de información y documentación clínica. BOE no. 274.

14. Villanueva E. En: Gisbert Calabuig JA, Villanueva Cañadas E, editores. Gisbert Calabuig. Medicina legal y toxicología. 6a ed. Barcelona: Masson; 2004.

15. Castellano M. La construcción de la Medicina Legal y Forense en España: después de ciento setenta años, una tarea inacabada. Publicación Academia Nacional de Medicina, 2012.

16. Committee on Identifying the Needs of the Forensic Sciences Community, National Research Council. Strengthening forensic science in the United States: A path forward. Document No.: 228091; 2009.

17. Milroy $C$. Full time forensic pathology service needs to be established. BMJ. 2001;323(7322):1183.

18. Griffith $\mathrm{R}$, Tengnah C. Proposals for legal reform in the coronial process 3. Br J Community Nurs. 2008;13(4):183-6.

19. DiMaio VJ. Medical examiners, forensic pathologists, and coroners. JAMA. 1997;277(7):531-2.

20. Jonas H. Técnica, medicina y ética. Barcelona: Paidós; 1997.

21. Amundsen DW. The physician's obligation to prolong life: a medical duty without classical roots. Hastings Cent Rep. 1978;8(4):23-30.

22. Wittgenstein L. Aforismos: cultura y valor. $2^{\mathrm{a}}$ ed. Madrid: Espasa Calpe; 1996. 